\title{
Distributed Control of Drinking Water Networks using Population Dynamics: Barcelona Case Study
}

\author{
J. Barreiro-Gómez, N. Quijano, Senior Member, IEEE and C. Ocampo-Martinez, Senior Member, IEEE
}

\begin{abstract}
Large-scale network systems involve a large number of variables, making the design of real-time controllers challenging. A distributed controller design allows to reduce computational requirements since tasks may be divided into different subsystems, making possible to guarantee real-time processing. This paper proposes a constrained optimizationbased distributed control design by applying a novel population and masses dynamics approach. The distributed controller design is applied to the Barcelona Drinking Water Network (DWN) in order to illustrate its effectiveness in the control performance.
\end{abstract}

\section{INTRODUCTION}

Network systems in engineering are usually large-scale problems, i.e., there is a big amount of variables in the system, becoming challenging to design real-time controllers owing to high computational costs. On the other hand, a centralized control requires full information about system states, which might imply additional communication issues when measuring the sensor signals (e.g., delays, packet losses). Drinking water management in urban areas has an increasing importance due to its influence in the supply of this indispensable resource due to social issues. Controllers for these systems are complex and should cope with constrained dynamical behaviors and operational criteria. Among constraints, there are limitations in elements in the network, e.g., tanks have their maximum storage capacity, the actuators have their physical restrictions to operate, the control action should be smooth to avoid damage in the network components, and the time-variant water demand must be satisfied. Additionally, it is necessary to minimize costs related to the energy required to operate valves and pumps, and the costs related to water that depend on proportions obtained from different sources available in the network. Therefore, this problem has many goals to weight up according to the importance assigned to each one. Consequently, control design based on constrained optimization may be convenient. That is why model predictive control (MPC) has been well studied and implemented in these systems [1][2]. A different approach to design decision-making systems

J. Barreiro-Gomez and N. Quijano are with Departamento de Ingeniería Eléctrica y Electrónica, Universidad de los Andes, Carrera $1^{\mathrm{A}}$ No $18 \mathrm{~A}-10$, Bogotá, Colombia \{j.barreiro135, nquijano\}@uniandes.edu.co.

J. Barreiro-Gomez and C. Ocampo-Martinez are with the Automatic Control Department, Universitat Politècnica de Catalunya, Institut de Robòtica Informàtica Industrial (CSIC-UPC), Llorens i Artigas, 4-6, 08028 Barcelona, Spain. \{jbarreiro, cocampo\}@iri.upc.edu.

This work has been partially supported by the projects COLCIENCIAS 548/2012, ECOCIS (Ref. DPI2013-48243-C2-1-R) and EFFINET (Ref. FP7-ICT-2011-8-31855). J. Barreiro-Gómez is supported by COLCIENCIAS and by the Agència de Gestió d'Ajust Universitaris i de Recerca AGAUR involving optimization concepts is game theory and the population dynamics approach [3]. The main characteristic to relate constrained optimization with game theory and population dynamics is that the Nash equilibria satisfy first order Karush-Kuhn-Tucker (KKT) conditions in full potential games [3]. Moreover, several distributed solutions for engineering problems have been proposed applying a game theoretical approach [4]. In [5], the replicator dynamics is applied to a dispatch problem in an optimal way. In [6], a game theoretical approach to solve constrained optimization problems is discussed.

The main contribution of this work is the design of a distributed control of Drinking Water Networks (DWN) by using a novel methodology based on population dynamics. This allows to have distributed intelligent systems in charge of a portion of the total amount of tasks, making more viable to guarantee real-time control. Moreover, the proposed method ensures that the feasible region is attractive, making the distributed control system robust against disturbances that could make conditions leave the feasible set. Finally, a distributed control for the Barcelona DWN designed with this methodology is presented.

The remainder of the paper is organized as follows. Section II presents some mathematical preliminaries regarding graphs and population dynamics. Section III describes the methodology proposed and shows stability analysis for the population and the masses dynamics; this section also presents the main constrained optimization problem solved with the proposed approach. Then, Section IV describes the Barcelona drinking water network, its states, constraints, and operational objectives. In Section V the results in simulation are presented, while in Section VI the main conclusions are drawn.

\section{GRAPHS AND POPULATION GAMES NOTATION}

The proposed decision-making system is oriented to control a network plant that is represented by an undirected connected graph denoted by $\mathcal{G}=(\mathcal{V}, \mathcal{E})$. This graph exhibits the topology of a society in a strategic interaction. The set of vertices or nodes denoted by $\mathcal{V}$, represents the control actions in the system and also corresponds to the set of strategies $S=\{1, \ldots, N\}$ in a strategic interaction. The set of edges or links given by $\mathcal{E}=\{(i, j): i, j \in \mathcal{V}\}$, represents the possible information sharing between states of the system associated to each node (control action) throughout the network.

The network system is composed by $M$ sub-systems. Each sub-system is represented by a complete sub-graph contained in $\mathcal{G}$, which is known as a clique. A clique represents also 
a population in the strategic interaction from the set $\mathcal{P}=$ $\{1, \ldots, M\}$, where there is full availability of information about system states. The sub-systems are not independent, e.g., sub-systems have coupled dynamics affecting each other or there are constraints involving elements from different sub-systems. The set of cliques is denoted by $\mathcal{C}=\left\{\mathcal{C}^{p}: p \in\right.$ $\mathcal{P}\}$, where $\mathcal{C}^{p}=\left(\mathcal{V}^{p}, \mathcal{E}^{p}\right)$, i.e., $\mathcal{V}^{p} \subset \mathcal{V}$ and $\mathcal{E}^{p} \subset \mathcal{E}$. In the strategic interaction $\mathcal{V}^{p}$ represents the $N^{p}$ strategies from the set $S^{p}=\left\{i: i \in \mathcal{V}^{p}\right\}$ of the population $p \in \mathcal{P}$.

The number of sub-systems containing a certain node $i \in$ $\mathcal{V}$ is given by a function denoted by $G(i)=\sum_{p \in \mathcal{P}} g(i, p)$ where $g(i, p)=1$, if $i \in \mathcal{V}^{p}$, and $g(i, p)=0$, otherwise. Since $\mathcal{G}$ is a non-complete connected graph, each clique associated to a sub-system shares at least one node with another clique. The nodes communicating various cliques are channels known as intersection nodes between subsystems. The set of intersection nodes in a subsystem (i.e., a population in the society $p \in \mathcal{P}$ ) is denoted by $\mathcal{I}^{p}=\{i \in$ $\left.\mathcal{V}^{p}: G(i)>1\right\}$, and the set of intersection nodes in the system (i.e., the society) is given by $\mathcal{I}=\cup_{p \in \mathcal{P}} \mathcal{I}^{p}$.

Throughout the paper, the set $\left\{p: i \in \mathcal{V}^{p}\right\}$ refers to all populations $p \in \mathcal{P}$ containing a node $i$, e.g., for a case with three populations whose set of nodes are given by $\mathcal{V}^{1}=$ $\{1,2\}, \mathcal{V}^{2}=\{2,3\}$ and $\mathcal{V}^{3}=\{3,4\}$, then $\left\{p: 2 \in \mathcal{V}^{p}\right\}=$ $\{1,2\}$ whereas $\left\{p: 4 \in \mathcal{V}^{p}\right\}=\{3\}$.

The scalar $x_{i}$ is the amount of agents in the society selecting the strategy $i \in S$, and the scalar $x_{i}^{p}$ is the amount of agents selecting the strategy $i \in S^{p}$ in the population $p \in \mathcal{P}$. The distribution of agents throughout the available strategies is known as the social state and the population state denoted by $x \in \mathbb{R}^{N}$ and $x^{p} \in \mathbb{R}^{N^{p}}$, respectively. For the strategic interaction, the set of social states (associated to control actions in the system) is given by a simplex denoted by $\Delta$, i.e., $\Delta=\left\{x \in \mathbb{R}_{+}^{N}: \sum_{i \in S} x_{i}=m\right\}$, where $m$ is the mass of agents in the society, i.e., an amount of a resource associated to actuators of the system. The set of population states (associated to control actions in a subsystem) is given by a simplex denoted $\Delta^{p}$ where $p \in \mathcal{P}$, i.e., $\Delta^{p}=\left\{x^{p} \in \mathbb{R}_{+}^{N^{p}}: \sum_{i \in S^{p}} x_{i}^{p}=m^{p}\right\}$, where $m^{p}$ is the mass of the population $p$, i.e., an amount of a resource associated to actuators at each sub-system.

There is a relationship between the social states (resource in the system) and the population states (resource in the subsystems), where $x_{i}^{p}=0$ if $i \notin \mathcal{V}^{p}$,

$$
x_{i}=\frac{1}{G(i)} \sum_{p \in \mathcal{P}} x_{i}^{p} .
$$

The resource could be the total energy applied to all the actuators in a system and/or a sub-system.

An actuator in the system affects directly a state of the system, then there is a function that depends on the state of the system, which determines the behavior of the associated actuator. These functions (known as fitness functions) determine how masses should be distributed among sub-systems (populations) within the system (society). Also, these fitness functions distribute masses among population states $\left(x_{i}^{p}\right.$ for all $i \in \mathcal{V}^{p}$ ) within each sub-system.
Remark 1: The population states and the social states are associated to the actuators and depend on the fitness functions which are associated to the states of the system, i.e., the control actions depend on states of the system as it is usual in a control system.

Let $F_{i}: \Delta \rightarrow \mathbb{R}$ be the fitness function for the proportion of agents playing strategy $i \in S$. The fitness function for the proportion of agents playing strategy $i \in S^{p}$ is $F_{i}^{p}: \Delta^{p} \rightarrow$ $\mathbb{R}$. The fitness for a strategy $i \in S$ is the same as the fitness for a strategy $j \in S^{p}$ if $i=j$. Consequently, for all $i \in S^{p}$ and for all $p: i \in \mathcal{V}^{p}$,

$$
F_{i}(x)=F_{i}^{p}\left(x^{p}\right), \text { if } x_{i}=x_{i}^{p}
$$

Society average function denoted $\bar{F}(x)$ is denoted by $\bar{F}(x)=$ $\frac{1}{m} \sum_{i \in S} x_{i} F_{i}$. The average function for all populations $p \in \mathcal{P}$ denoted $\bar{F}^{p}\left(x^{p}\right)$ is $\bar{F}^{p}\left(x^{p}\right)=\frac{1}{m^{p}} \sum_{i \in S^{p}} x_{i}^{p} F_{i}^{p}$. The method proposed in this paper, is restricted to the assumptions below.

Assumption 1: The game $F(x)$ is a full potential game, i.e., there is a function $f(x)$ known as potential function such that the fitness functions are given by $F(x)=\frac{\partial f(x)}{\partial x}$. In this kind of games, the Nash equilibrium denoted $x^{*}$ corresponds to an extreme point of the potential function $f(x)$ satisfying the Karush-Kuhn-Tucker (KKT) first order conditions [3]. $\diamond$

Assumption 2: The game $F(x)$ is stable, i.e., the potential function $f(x)$ is concave.

Assumption 3: Fitness functions in a sub-system only depend on elements that belong to the same sub-system. i.e., the required information for a decision-making system is available in the sub-system.

Assumption 4: The population masses are strictly positive, i.e., $m^{p}>0$, for all $p \in \mathcal{P}$; and there is not extinction of proportion of agents playing a strategy, i.e., the scalar $x_{i}^{p}>0$, for all $p \in \mathcal{P}$, and $i \in S^{p}$.

\section{DYNAMICS FOR SUB-SYSTEMS AND INTERSECTION NODES}

The objective for the system (society) is to establish optimal social states related to the actuators in the system according to a potential function $f(x)$ that depends on the states of the system. Consequently, the social states converge to a Nash equilibrium denoted $x^{*} \in \Delta$, which implies that in mixed-strategies $F_{i}=F_{j}$ for all $i, j \in S$.

The goal is achieved by using sub-systems dynamics and intersection nodes dynamics. At each sub-system (population), there is a game converging to a Nash equilibrium denoted $x^{p *} \in \Delta^{p}$ implying that $F_{i}^{p}=F_{j}^{p}$ for all $i, j \in$ $S^{p}$, and each intersection node has dynamics allowing an interchange of masses among populations.

\section{A. Dynamics for sub-systems}

A game is solved for each sub-system (population) with constraints given by masses $m^{p}$ which vary dynamically. There are $M$ system dynamics of this type, one for each sub-system (clique) $\mathcal{C}^{p}$, for all $p \in \mathcal{P}$. Moreover,

$$
\dot{x}_{i}^{p}=x_{i}^{p}\left(F_{i}^{p}-\bar{F}^{p}-\phi^{p}\right), \text { for all } i \in S^{p},
$$




$$
\phi^{p}=\beta\left(\frac{1}{m^{p}} \sum_{j \in S^{p}} x_{j}^{p}-1\right)
$$

and $\beta$ is a convergence factor. Note that when $\phi^{p}=0$ (i.e., $x^{p} \in \Delta^{p}$ ) the differential equation in (3) is the replicator dynamics equation [3].

\section{B. Dynamics for intersection nodes}

On the other hand, there are dynamics for population masses $m^{p}$. The masses associated to each sub-system are varying depending on the intersections nodes behavior. There are dynamics of this type for all nodes $i \in \mathcal{I}$ such that

$$
\dot{m}_{i}^{p}=m_{i}^{p}\left(x_{i}-x_{i}^{p}\right) \text {, for all } p: i \in \mathcal{V}^{p} \text {. }
$$

There is a relationship between $m_{i}^{p}$ and $m^{p}$, $m^{p}=\frac{1}{\left|\mathcal{I}^{p}\right|} \sum_{i \in \mathcal{I}^{p}} m_{i}^{p}$, where $\left|\mathcal{I}^{p}\right|$ is the cardinality of the set $\mathcal{I}^{p}$. For these dynamics, column vectors of masses are defined for all $i \in \mathcal{I}$ as $\mathrm{m}_{i}=\left\{m_{i}^{p}\right.$, for all $\left.p: i \in \mathcal{V}^{p}\right\}$, $\mathrm{m}_{i} \in \mathbb{R}^{G(i)}$ and column vectors of population states for all $i \in \mathcal{I}$ as $\mathrm{x}_{i}=\left\{x_{i}^{p}\right.$, for all $\left.p: i \in \mathcal{V}^{p}\right\}, \mathrm{x}_{i} \in \mathbb{R}^{G(i)}$. Note that $\mathrm{m}_{i} \neq m_{i}$ and $\mathrm{x}_{i} \neq x_{i}$. The equilibrium point $\mathrm{m}_{i}^{*}$ implies that $x_{i}=x_{i}^{p}$, for all $p: i \in \mathcal{V}^{p}$. Consequently, the dynamics for the sub-systems converge to a Nash equilibrium, and the society satisfies an arbitrary simplex $\Delta$.

Proposition 1: If Assumption 4 is satisfied, the subsystems dynamics are in equilibrium $x^{p *} \in \Delta^{p}$, for all $p \in$ $\mathcal{P}$, and the intersection nodes dynamics are in equilibrium $\mathrm{m}_{i}^{*}$, for all $i \in \mathcal{I}$, then the system is in equilibrium $x^{*}$ (i.e., $F_{i}(x)=F_{j}(x)=\bar{F}(x)$, for all $i, j \in S$ ).

Proof: The equilibrium $x^{p *} \in \Delta^{p}$ of the sub-systems dynamics (3) for all $p \in \mathcal{P}$ implies that

i) $\phi^{p}\left(x^{p *}\right)=0$ and,

ii) $F_{i}^{p}\left(x^{p *}\right)=\bar{F}^{p}$, for all $i \in S^{p}$, and $p \in \mathcal{P}$.

The equilibrium $\mathrm{m}_{i}{ }^{*}$ of the intersection nodes dynamics (4), for all $i \in \mathcal{I}$ implies that $x_{i}^{*}=x_{i}^{p^{*}}$ for all $p: i \in \mathcal{V}^{p}$. Then, for all $i \in \mathcal{I}$, it holds that $x_{i}^{*}=x_{i}^{r *}=x_{i}^{k^{*}}$ for all $r$ : $i \in \mathcal{V}^{r}$ and $k: i \in \mathcal{V}^{k}$. By (2) $F_{i}\left(x^{r *}\right)=F_{i}\left(x^{k^{*}}\right)=F_{i}\left(x^{*}\right)$. Moreover, $F_{i}\left(x^{r *}\right)=\bar{F}^{r}\left(x^{r *}\right)=F_{i}\left(x^{k^{*}}\right)=\bar{F}^{k}\left(x^{k^{*}}\right)$ for all $r, k \in \mathcal{P}$ and $i \in \mathcal{I}$. Consequently, all population average fitnesses are equal, then $F_{i}\left(x^{*}\right)=F_{j}\left(x^{*}\right)=\bar{F}\left(x^{*}\right)$ for all $i, j \in S$, which completes the proof.

In order to have control on the solution of the strategic interaction, it is convenient to achieve the equilibrium in an imposed/desired value for the fitness functions. The desired value is a reference denoted by $R$. Then, the dynamics would establish the appropriate masses so that the average fitness coincides with the reference at the Nash equilibrium point. In order to guarantee the convergence of the fitness functions to $R$, it is ensured that $x_{i}^{p}$ approaches to the known value $\tilde{x}_{i}$, for all $p: i \in \mathcal{V}^{p}$. Additionally, it is known that $F_{i}\left(\tilde{x}_{i}\right)=R$ for any $i \in \mathcal{I}$. The desired value is forced by modifying the relationship in (1) only for one intersection node $i \in \mathcal{I}$ as $x_{i}=\frac{1}{G(i)+1} \sum_{p \in \mathcal{P}} x_{i}^{p}+\tilde{x}_{i}$.
Remark 2: In case that $\tilde{x}_{i}$ is not easily found for any $i \in \mathcal{I}$ so that $F_{i}\left(\tilde{x}_{i}\right)=R$. It is possible to establish any decreasing fictitious function denoted by $\tilde{F}\left(x_{N+1}\right)$, where $x_{N+1}$ is an intersection node, and $\tilde{x}_{N+1}$ is known so that $\tilde{F}\left(\tilde{x}_{N+1}\right)=R$. The addition of a new variable does not affect the solution of the whole problem, but allows to force the trajectories to converge to the desired value $R$.

The structure corresponding to a case with three cliques and an intersection node is presented in Figure 1.



Fig. 1. Structure of dynamical system for a system with three subsystems and one intersection node.

\section{Stability Analysis}

It is shown that the equilibrium for both dynamics (i.e., the ones for sub-systems and the ones for intersection nodes) are asymptotically stable.

Theorem 1: If $F(x)$ is a stable game, then there exists a $\beta$ such that the equilibrium point $x^{p *} \in \Delta^{p}$ of (3) for all $p \in \mathcal{P}$, and the equilibrium point $\mathrm{m}_{i}{ }^{*}$ of the masses dynamics in (4) for all $i \in \mathcal{I}$, are asymptotically stable.

Proof: Using the convex radially unbounded Lyapunov function $V\left(x^{p}, \mathrm{~m}_{i}\right)^{1}$,

$$
\begin{array}{r}
V\left(x^{p}, \mathrm{~m}_{i}\right)=\sum_{p \in \mathcal{P}} \sum_{i \in S^{p}} x_{i}^{p}-x_{i}^{p^{*}}\left(1+\ln \left(\frac{x_{i}^{p}}{x_{i}^{p^{*}}}\right)\right)+ \\
\sum_{i \in \mathcal{I}} \sum_{p: i \in \mathcal{V}^{p}} m_{i}^{p}-m_{i}^{p *}\left(1+\ln \left(\frac{m_{i}^{p}}{m_{i}^{p^{*}}}\right)\right)
\end{array}
$$

where, $V\left(x^{p *}, \mathrm{~m}_{i}{ }^{*}\right)=0$, and $V\left(x^{p}, \mathrm{~m}_{i}\right)>0$ if $x^{p} \neq x^{p *}$ or $\mathrm{m}_{i} \neq \mathrm{m}_{i}{ }^{*}$. Its derivative considering one population and one intersection node is

$$
\dot{V}\left(x^{p}, \mathrm{~m}_{i}\right)=\sum_{i \in S^{p}}\left(1-\frac{x_{i}^{p *}}{x_{i}^{p}}\right) \dot{x}_{i}^{p}+\sum_{p: i \in \mathcal{V}^{p}}\left(1-\frac{m_{i}^{p *}}{m_{i}^{p}}\right) \dot{m}_{i}^{p} .
$$

Make a change of variable $\mathbb{1} x_{i}^{\top} \mathbb{1} \frac{1}{G(i)}=\tilde{x}_{i}$, where $\mathbb{1}=$ $\left[\begin{array}{llll}1 & 1 & \ldots & 1\end{array}\right]^{\top}$ is a column of ones with suitable dimensions for population states (i.e., its cardinality is $|\mathbb{1}|=N^{p}$ ) and for masses (i.e., its cardinality is $|\mathbb{1}|=G(i)$ ). Also, it is considered that $x^{p^{\top}} \mathbb{1}=m^{p}+\epsilon$ where $\epsilon$ could be either positive or negative depending on whether $x^{p}$ is geometrically either above or below the simplex $\Delta^{p}$, then

\footnotetext{
${ }^{1}$ Details of this proof have been omitted due to the lack of space.
} 


$$
\begin{aligned}
\dot{V}\left(x^{p}, \mathrm{~m}_{i}\right)= & \alpha x^{p \top} F\left(x^{p}\right)-\beta \frac{\epsilon^{2}}{m^{p}}+\left(x^{p}-x^{p *}\right)^{\top} F\left(x^{p}\right)+ \\
& \left(\mathrm{m}_{i}^{*}-\mathrm{m}_{i}\right)^{\top}\left(\mathrm{x}_{i}-\tilde{\mathrm{x}}_{i}\right) .
\end{aligned}
$$

with $\alpha=\left(1-\frac{m^{p}+\epsilon}{m^{p}}\right)$. There exists a $\beta$ such that $\dot{V}\left(x^{p}, \mathrm{~m}_{i}\right) \leq 0$ given by

$$
\begin{aligned}
\beta \geq & \frac{m^{p}}{\epsilon^{2}}\left\{\left(1-\frac{m^{p}+\epsilon}{m^{p}}\right) x^{p^{\top}} F\left(x^{p}\right)+\left(x^{p}-x^{p *}\right)^{\top} F\left(x^{p}\right)+\right. \\
& \left.\left(\mathrm{m}_{i}^{*}-\mathrm{m}_{i}\right)^{\top}\left(\mathrm{x}_{i}-\tilde{\mathrm{x}}_{i}\right)\right\} .
\end{aligned}
$$

Note that when population states are near a feasible region (i.e., $\epsilon \rightarrow 0$ ), the convergence factor is not longer required (i.e., $\beta$ can get any positive value or zero).

Remark 3: This theorem also shows that the feasible region is attractive, i.e., the trajectories converge to a feasible region when the population states are out of it or when disturbances force the state to leave this set.

\section{Optimization problem with constraints}

Since a Nash equilibrium in a potential game satisfies the Karush-Kuhn-Tucker (KKT) first order condition, an optimization problem in a distributed way can be solved by applying the population dynamics and the masses dynamics introduced in Sections III-A and III-B, respectively.

Suppose that we want to design a decision-making system to control (in a distributed way) a network system composed by various sub-systems. The desired behavior for the network is given by a constrained optimization problem, i.e.,

$$
\max f(x) \text {, s. t. } A x=b \text {, and } x_{i} \geq 0,1 \leq i \leq N \text {, }
$$

where $x \in \mathbb{R}_{+}^{N}, f: \mathbb{R}_{+}^{N} \rightarrow \mathbb{R}$. It is assumed that $f(x)$ is continuously differentiable, $f \in C^{1}$, and concave. $A \in \mathbb{R}^{V \times N}$ since there are $V$ constraints and $N$ decision variables, and $b \in \mathbb{R}^{V}$. For this constrained optimization problem, $\mu$ is the Lagrange multiplier vector and the Lagrange function $l: \mathbb{R}^{N} \times \mathbb{R}^{V} \rightarrow \mathbb{R}$ is $l(x, \mu)=f(x)+\mu^{\top}(A x-b)$, where $\nabla_{x} l(x, \mu)=\nabla f(x)+A^{\top} \mu$, and $\nabla_{\mu} l(x, \mu)=-A x+b$.

The Lagrange condition is used to find possible extreme points in a cost function. In the optimal point $\nabla_{x} l\left(x^{*}, \mu^{*}\right)=$ 0 and $\nabla_{\mu} l\left(x^{*}, \mu^{*}\right)=0$. Consequently, new nodes $\mu_{i}$ for constraints are added. The fitness functions for each node are chosen as $F(x)=\nabla_{x} l(x, \mu)$ and $F(\mu)=\nabla_{\mu} l(x, \mu)$. The problem is solved imposing a reference value $R=0$ by applying a fictitious function as highlighted in Remark 2.

\section{CAse Study Application: Barcelona DWN}

The Barcelona DWN, managed by Aguas de Barcelona S.A (AGBAR), supplies drinking water to Barcelona city and its metropolitan areas. The network has a centralized telecontrol system, organized in a two-level architecture. At the upper level, a supervisory control system, installed in the control centre of AGBAR, is in charge of controlling the whole network by taking into account operational constraints and consumer demands. This upper level provides the setpoints for the lower-level control system. The lower level optimizes the pressure profile to minimize losses due to leakage and to provide sufficient water pressure, e.g., for high-rise buildings. For more details of this system, see [7].

\section{A. System management criteria}

In order to control the DWN, there are two main criteria for a desired performance.

1) Minimize operational costs associated to water supply and energy for water transport: Economic costs associated to drinking water production are generated by chemicals for treatment water, legal canons, and active elements electrical costs (pumps). The function to minimize is given by $f_{1}(t)=W_{c}\left(\alpha_{1}+\alpha_{2}(t)\right) u$, where $W_{c}$ establishes a weight for a total cost function, $u \in \mathbb{R}^{N}$ denotes the flows that can be manipulated, $\alpha_{1}$ is a known column vector whose cardinality is $N$ associated to water costs according to the corresponding source (treatment plant, dwell, etc.). $\alpha_{2}(t)$ is a column vector whose cardinality is $N$ describing operation energy costs associated to pumps, which varies over time since electricity costs have different values during the day.

2) Ensure safety storage terms, which are related to satisfy demand at each point of the network: Satisfaction of water demands should be met all the time and some risk prevention mechanism should be considered as a storage of water volume availability of emergency for future demands given by a relation respect to the maximum volume at each tank $\xi v^{\max }$. The cost function to minimize is $f_{2}(t)=(v-$ $\left.\xi v^{\max }\right)^{\top} W_{v}\left(v-\xi v^{\text {max }}\right)$, where $v \in \mathbb{R}^{V}$ denotes the water volumes at network tanks, $\xi$ is a term that determines the safety volume to be considered with respect to the maximum volume tank vector $v^{\max } \in \mathbb{R}^{V}$. $W_{v}$ is a weight matrix that ponderates the total objective function.

\section{B. DWN Model and constraints}

The DWN can be considered as a large-scale system. Figure 2 shows the DWN in Barcelona. The control system presented in this paper manages the transport network problem and sets references for local PID controllers, which are in a lower level of the hierarchical structure. DWN is a non-linear system; however, for management level, it is considered linear due to the fact that non-linearities are considered in the low level controllers.

The mass balance expression relating the stored volume $v \in \mathbb{R}^{V}$, the manipulated flows, and demand flows can be written as $\dot{v}_{i}=\sum_{n} q_{(\text {in }), n}(t)-\sum_{m} q_{(\text {out }), m}(t)$, where, for all time $t, q_{(\text {in }), n}(t)$ and $q_{(\text {out }), m}(t)$ are the $n^{\text {th }}$ inflow and the $m^{\text {th }}$ outflow related to the $i^{t h}$ tank, respectively. These flows are given in $\left[\mathrm{m}^{3} / \mathrm{s}\right]$.

There are physical constraints for the tanks according to their capacity, i.e., $v_{i}^{\text {min }} \leq v_{i}(t) \leq v_{i}^{\max }$, where $v_{i}^{\text {min }}$ is the minimum volume capacity and $v_{i}^{\max }$ is the maximum capacity given in $\left[\mathrm{m}^{3}\right]$. Then, it is not possible to assign more water to a filled tank or drain water from an empty tank. Regarding the control actions $u_{i}(t)$, there are two types of actuators: pumps and valves.

These elements have physical constraints as well, i.e., $u_{i}^{\min } \leq u_{i}(t) \leq u_{i}^{\max }$, where $u_{i}^{\min }$ and $u_{i}^{\max }$ denote the minimum and maximum flow capacity, respectively, given in $\left[\mathrm{m}^{3} / \mathrm{s}\right]$. Figure 2 shows 11 nodes in the network, where the mass balance should be respected, adding more constraints to the problem. 


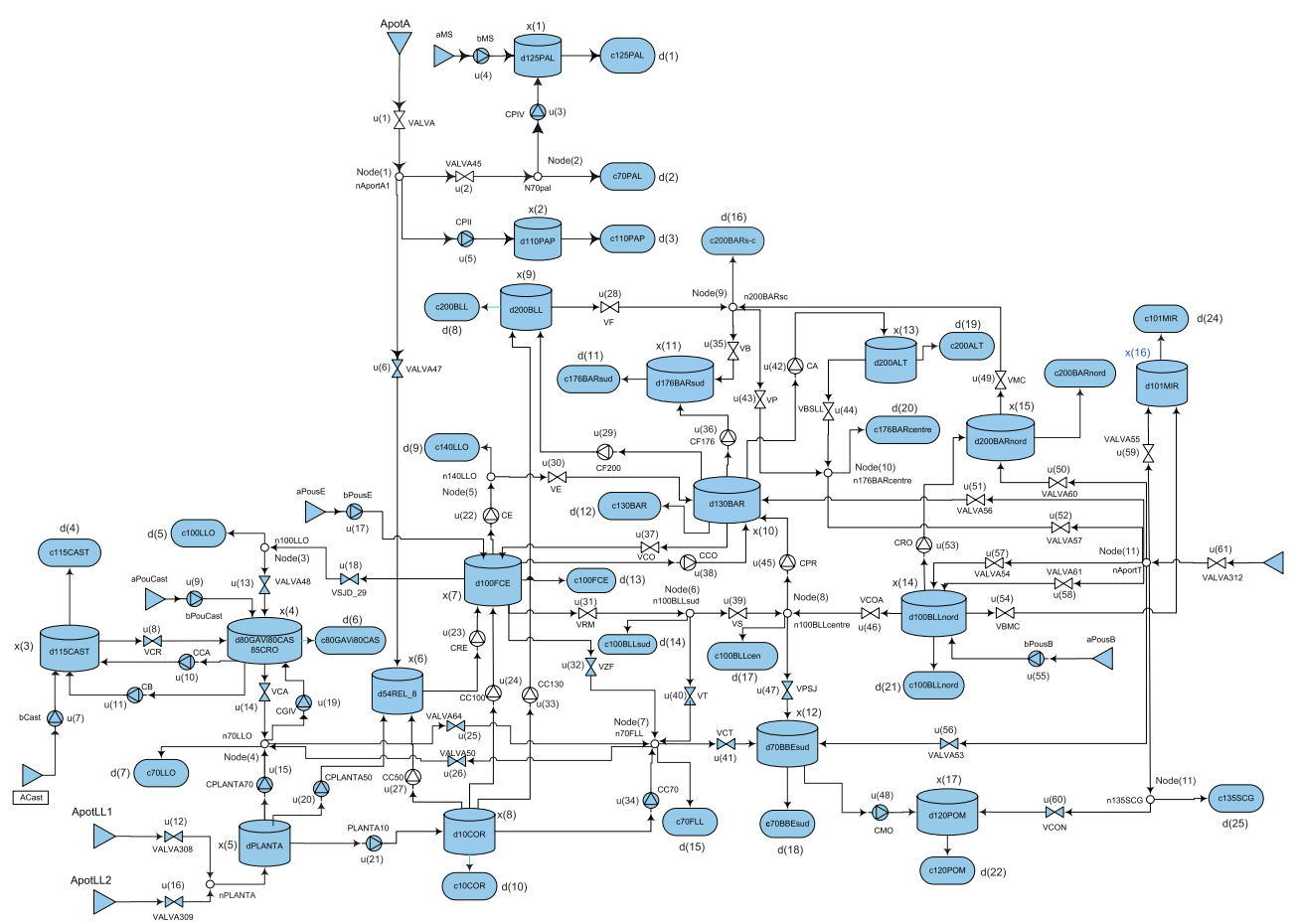

Fig. 2. Barcelona drinking water network. Partition of the network into three cliques according to Table II.

TABLE I

NODE CONSTRAINTS

\begin{tabular}{cl}
\hline Node & Constraint \\
\hline 1 & $u(1)-u(2)-u(5)-u(6)=0$ \\
2 & $u(2)-u(3)=d(2)$ \\
3 & $u(18)-u(13)=d(5)$ \\
4 & $u(14)+u(26)+u(15)-u(19)-u(25)=d(7)$ \\
5 & $u(22)-u(30)=d(9)$ \\
6 & $u(31)-u(40)-u(39)=d(14)$ \\
7 & $u(32)+u(40)+u(34)+u(25)-u(41)-u(26)=d(15)$ \\
8 & $u(39)+u(46)-u(45)-u(47)=d(17)$ \\
9 & $u(28)+u(49)-u(35)-u(43)=d(16)$ \\
10 & $u(44)+u(43)+u(52)=d(20)$ \\
11 & $u(61)-u(59)-u(50)-u(51)-u(52)-u(57)-u(58)$ \\
& $-u(56)-u(60)=d(25)$ \\
\hline
\end{tabular}

The constraints are expressed as $\sum_{n} q_{(i n), n}(t)=$ $\sum_{m} q_{(\text {out }), m}(t)$, where $q_{(\text {in }), n}(t)$ is the $n^{t h}$ the node inflow and $q_{\text {(out }), m}(t)$ is the $m^{t h}$ node outflow. Some of these flows are manipulated as part of the control actions, and others are set by water demands. Moreover, demands can be forecasted and they are assumed to be known, where $d$ is a vector with measured disturbances affecting DWN. Node constraints are explicitly expressed in Table I.

\section{Partitioning of the network}

Partitioning of the DWN is a problem already studied in [8]. For the DWN control problem, partitioning is determined based on system constraints. As in Section III-D, Lagrange multiplier vertices are connected to vertices from which information is needed to set the respective constraint and these Lagrange multipliers must belong to the same clique. Based on this idea, it is possible to determine vertices (strategies) that should belong to the same clique (population). For example, the constraint given by node 9 shares a vertex with constraint given by node 10 , this vertex is $u_{43}$; however, constraints for nodes 10 and 11 involve vertex $u_{52}$. Consequently, there is a clique including all elements involved in constraints 9, 10, and 11 from Table I. In the same way, vertices corresponding to the slack variable $s(j)$ should belong to the same clique to which vertex $u_{j}$ belongs. On the other hand, there are some vertices that are not associated to any constraint, e.g., $u_{4}$ or $u_{55}$. In these cases, the vertices are assigned to the nearest clique. Cliques are presented in Table II.

\section{TABLE II}

PARTITION OF THE NETWORK INTO CLIQUES

\begin{tabular}{cl}
\hline Clique & Vertices $u, s \in \mathcal{V}^{p}$ \\
\hline$p=1$ & $1,2,3,4,5,6,7,8,9,10,11,13,17,18,22,29,30,36,37,38$ \\
$p=2$ & $12,14,15,16,19,20,21,23,24,25,26,27,31,32,33,34,39$, \\
& $40,41,45,46,47$ \\
$p=3$ & $28,35,42,43,44,48,49,50,51,52,53,54,55,56,57,58,59$, \\
& 60,61
\end{tabular}

\section{Optimization Problem}

The controller is designed as an optimization problem minimizing costs and the error with respect to the safety storage term subject to physical constraints of nodes, tanks, and actuators. To this end, a new variable $\tilde{v}(t) \in \mathbb{R}^{N}$ is introduced, where $\tilde{v}_{i}(t)$ is the volume corresponding to the tank whose input flow is given by $u_{i}(t)$, i.e., $\tilde{v}_{i}(t)=v_{j}(t)$ if $u_{i}(t)$ is the input flow of the $j^{t h}$ tank. In case that the control action $u_{i}$ is not an input flow to any tank, then $\tilde{v}_{i}=0$. In the same way, $\tilde{v}^{\max } \in \mathbb{R}^{N}$ corresponds to the maximum volume, e.g., for the control action $u_{4}(t), \tilde{v}_{4}(t)=v_{1}(t)$, since $u_{4}(t)$ is the input flow for tank 1 , and the maximum volume $\tilde{v}_{4}^{\max }=v_{1}^{\max }$. For the control action $u_{2}(t), \tilde{v}_{2}(t)=0$ since $u_{2}(t)$ is not an input flow for any tank, and the maximum volume $\tilde{v}_{2}^{\max }=0$ for the same reason. The error is defined as $e(t)=\left(\xi \tilde{v}^{\max }-\tilde{v}\right), e(t) \in \mathbb{R}^{N}$ since it is known that error $e(t)$ is reduced as $u(t)$ increases, assigning more drinking 
water to tanks with more error. The scalar $e_{i}(t)=\xi \tilde{v}_{i}^{\max }-\tilde{v}_{i}$ is the error of the tank for which $u_{i}$ is its input flow. The error $e_{i}(t)=\xi \tilde{v}_{i}^{\max }-\tilde{v}_{i}=0$, if $u_{i}$ is not an inflow for any tank. The optimization problem is given by

$$
\begin{aligned}
\max f(u)= & -W_{v}\left(\tilde{v}-\xi \tilde{v}^{\max }\right)^{\top} \operatorname{diag}(u(t))\left(\tilde{v}-\xi \tilde{v}^{\max }\right) \\
& -W_{c}\left(\alpha_{1}+\alpha_{2}(t)\right) u(t) \\
\text { s. t. } & A u=b, \quad I u \leq c
\end{aligned}
$$

where $A \in \mathbb{R}^{11 \times 61}$ is a matrix determined by the constraints presented in Table I corresponding to the nodes, $I \in \mathbb{R}^{61 \times 61}$ is the identity matrix fixing constraints related to actuators, $b=\left[\begin{array}{llllll}0 & d_{2} & d_{5} & d_{7} & \ldots & d_{25}\end{array}\right]^{\top}, b \in \mathbb{R}^{11}$ (see Figure 2), and $c=\left[\begin{array}{llll}u_{1}^{\max } & u_{2}^{\max } & \ldots & u_{N}^{\max }\end{array}\right]^{\top}, c \in \mathbb{R}^{61}$. Then, consider the vector of states $\tilde{x} \in \mathbb{R}^{122}$ with the slack variables $s$ given by $\tilde{x}=\left[u^{\top}, s^{\top}\right]^{\top}$, and two new matrices $\tilde{A}$ and $\tilde{b}$ as

$$
\tilde{A}_{72 \times 122}=\left[\begin{array}{c|c}
A_{11 \times 61} & 0_{11 \times 61} \\
\hline I_{61 \times 61} & I_{61 \times 61}
\end{array}\right], \quad \tilde{b}_{72}=\left[\begin{array}{c}
b_{11} \\
\hline c_{61}
\end{array}\right] \text {. }
$$

The new optimization problem has the form as in (5) and it can be solved using population dynamics, whose fitnesses are the Lagrangian function, i.e.,

$$
\max f(\tilde{x}) \text {, s. t. } \tilde{A} \tilde{x}=\tilde{b} \text {, and } \tilde{x}_{i} \geq 0,1 \leq i \leq 122 \text {. }
$$

This problem is solved by applying the dynamics introduced in Section III in a distributed way, and satisfying all constraints established for the DWN.

\section{Results}

Simulations consider the following parameters: demand requirements establishes a $\xi=0.2$, and weight for functions are selected to assign more importance to the minimization of costs, then $W_{c}=10$ and $W_{v}=0.1$. Initial conditions for simulation are chosen arbitrarily with respect to the maximum volume, i.e., $v(0)=0.386 v^{\max }$. Results for the distributed optimization based controller with population dynamics are compared with results obtained with a distributed model predictive control (DMPC) presented in [1]. Evolution of the DWN states of tanks 14 and 15 have been chosen to be analyzed since they involve a considerable number of control actions $\left(u_{50}, u_{53}-u_{55}, u_{57}, u_{58}, u_{61}\right)$, water sources and demand points (c200BARnord, C100BLLnord), see Figure 2. The evolution of volumes at tanks 14 and 15 are shown in Figure 3. Volumes oscillate around the set point despite the hard disturbances given by demands.

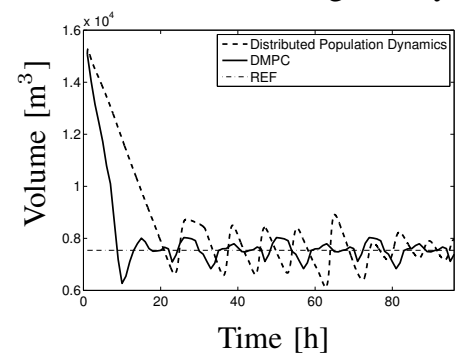

a)

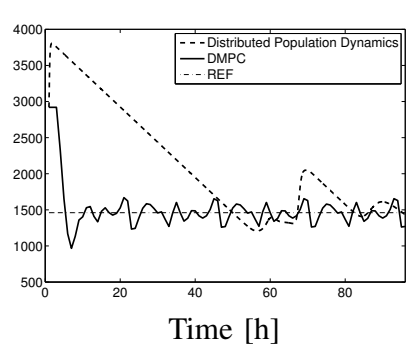

b)
Fig. 3. Volume evolution with reference for tanks a) 14 and b) 15 .

The behavior is oscillatory due to the periodicity of the water demand (system disturbances), yielding in a controller more focused on disturbance rejection instead of regulation or tracking problems. It is important to highlight that results along the first days are not representative due to the fact that the system initial conditions do not correspond to the periodical behavior of the system.

Regarding water and energy costs related to the operation of actuators, data are discriminated in Table III during four

\begin{tabular}{|c|c|c|c|c|c|c|}
\hline \multirow[b]{2}{*}{ Day } & \multicolumn{3}{|c|}{$\overline{\text { DMPC }}$} & \multicolumn{3}{|c|}{ WITH POPUL. DYNAMICS } \\
\hline & Water & Electric & Total & Water & Electric & Total \\
\hline 1 & 21.56 & 21.94 & 43.50 & 24.07 & 16.88 & 40.95 \\
\hline 2 & 27.67 & 21.90 & 49.57 & 29.46 & 19.14 & 48.60 \\
\hline 3 & 27.57 & 21.94 & 49.51 & 30.12 & 26.79 & 56.91 \\
\hline 4 & 27.58 & 21.94 & 49.52 & 30.13 & 26.71 & 56.84 \\
\hline
\end{tabular}
days $^{2}$ for both controllers, DMPC and distributed controller based on population dynamics.

TABLE III

DISCRIMINATION OF ECONOMIC COSTS

\section{CONCLUSIONS}

A distributed model-free control has been designed by applying the proposed methodology based on population dynamics for subsystems and dynamics associated to intersection nodes among subsystems in a DWN, particularly in the Barcelona case study. This optimization-based controller considers the multiple constraints associated to tanks, actuators and demand, and a minimization of costs of water and energy. Results show that, despite the critic disturbances, volumes in the system tend to their corresponding setpoints minimizing the difference between current states and safety storage terms. On the other hand, the methodology allows to have a decision-making system at each subsystem distributing tasks, and this distribution does not affect the consideration of constraints for the whole system.

\section{REFERENCES}

[1] C. Ocampo-Martinez, V. Fambrini, D. Barcelli, and V. Puig, "Mode predictive control of drinking water networks: A hierarchical and decentralized approach," in In Proceedings of the American Control conference (ACC), 2010., pp. 3951-3956.

[2] C. Ocampo-Martinez, V. Puig, G. Cembrano, and J. Quevedo, "Application of predictive control strategies to the management of complex networks in the urban water cycle," IEEE Control Systems Magazine, vol. 33, no. 1, pp. 15-41, 2013.

[3] W. H. Sandholm, Population games and evolutionary dynamics, ser. Economic learning and social evolution. Cambridge, Mass. MIT Press, 2010 .

[4] J. Marden and J. Shamma, "Game theory and distributed control," in Handbook of Game Theory Vol. 4, H. Young and S. Zamir, Eds. Elsevier Science, 2013.

[5] A. Pantoja and N. Quijano., "Distributed optimization using population dynamics with a local replicator equation." in Proceedings of the IEEE Conference on Decision and Control (CDC), 2012, pp. 3790-3795.

[6] N. Li and J. R. Marden, "Designing games for distributed optimization," IEEE Journal of Selected Topics in Signal Processing, vol. 7, no. 2, pp. 230-242, 2013, special issue on adaptation and learning over complex networks.

[7] C. Ocampo-Martinez, V. Puig, G. Cembrano, R. Creus, and M. Minoves, "Improving water management efficiency by using optimization-based control strategies: the Barcelona case study," Water Science \& Technology: Water Supply, vol. 9, no. 5, pp. 565-575, 2009.

[8] C. Ocampo-Martinez, S. Bovo, and V. Puig, "Partitioning approach oriented to the decentralised predictive control of large-scale systems," Journal of Process Control, vol. 21, pp. 775-786, 2011.

\footnotetext{
${ }^{2}$ These costs are expressed in economic units instead of in the real value (Euro) because of confidentiality reasons.
} 